\title{
Beethoven como instrumento narrativo en la novela de la primera mitad del siglo $\mathrm{XX}$
}

\author{
Beethoven as a narrative instrument \\ in the novel of the first half of the $20^{\text {th }}$ century
}

Juan José Pastor Comín

Universidad de Castilla-La Mancha

Centro de Investigación y Documentación Musical (CIDoM)-Unidad Asociada al CSIC juanjose.pastor@uclm.es ORCID iD: https://orcid.org/0000-0001-8165-1232

\section{RESUMEN}

Los estudios de recepción musical suelen centrarse tanto en la influencia de las músicas del pasado sobre las composiciones que les sucedieron, así como sobre el grado de ejecución y presencia en la vida cultural de los distintos presentes. No existen, sin embargo, estudios que analicen con detalle cómo tanto la música como la figura personal de cada compositor perviven en otras manifestaciones artísticas que seleccionan, amplifican, manipulan o se apropian, bajo las coordenadas de sus propias disciplinas, de la obra musical heredada. Este artículo profundizará en cómo Beethoven y su música fue un poderoso instrumento en la construcción de algunas de las obras literarias más relevantes de la primera mitad de siglo a través de autores como Romain Rolland, Proust, E. M. Forster, James Weldon Johnson, Virginia Woolf, Aldous Huxley, Malraux o Thomas Mann, atendiendo tanto a su aprovechamiento técnico, constructivo, semántico, social, como, sin duda, estético.

Palabras Clave: Música y literatura, transmedialidad, interdisciplinariedad, Beethoven, recepción musical. 


\title{
JUAN José PASTOR COMÍN
}

\begin{abstract}
Studies of musical reception tend to focus both on the influence of the music of the past on the compositions that followed them, as well as on the degree of performance and presence in the cultural life of the different presents. There are, however, no studies that analyze in detail how both music and the personal figure of each composer survive in other artistic manifestations that select, amplify, manipulate or appropriate, under the coordinates of their own disciplines, the inherited musical work. This article will delve into how Beethoven and his music were a powerful instrument in the construction of some of the most relevant literary works of the first half of the century through authors such as Romain Rolland, Proust, EM Forster, Virginia Woolf, James Weldon Johnson, Aldous Huxley, Malraux or Thomas Mann, taking into account both its technical, constructive, semantic, social, and, without a doubt, aesthetic use.
\end{abstract}

Key Words: Music and literature, transmediality, interdisciplinarity, Beethoven, musical reception.

Pastor Comín, J. J. (2020). Beethoven como instrumento narrativo en la novela de la primera mitad del siglo XX. Cuadernos de Investigación Musical, (11, número extraordinario), pp. 195-215.

\section{INTRODUCCIÓN}

La poderosa figura de Beethoven y su música han encontrado un lugar más allá de la esfera específicamente musical, proyectándose con fuerza sobre otros ámbitos artísticos tales como las artes plásticas o la literatura. Este camino paralelo por el que discurre la recepción y percepción tanto de su persona - deturpada por los barnices mitógrafos barnices románticos- como de su propia obra -fuera del ámbito técnico-compositivo- ha sido, sin embargo, muy poco transitado y atendido (Capdepón \& Pastor, 2020), a pesar de que el resto de las artes ofrecen testimonios doblemente reveladores: demuestran, por un lado, cómo ha sido entendido y asimilado el patrimonio musical beethoveniano y su figura emblemática, acogiendo una serie de sentidos y valores que se actualizan sobre cada presente con finalidades distintas; por otro, nos informan de cómo constituyen una semilla fecunda que contribuye a la creación de nuevas obras artísticas - en nuestro caso literarias- gracias a su condición de referente inexcusable, tanto estructural como referencial y semántico. Si bien hemos tratado en otras publicaciones con exhaustividad la presencia de Beethoven en la poesía universal (Pastor, 2020a), nos centraremos aquí en algunos usos significativos de su música en las figuras más relevantes de la eclosión de la novela moderna, que adoptaron servirse no tanto del como figura emblemática de nuestro compositor sino de su obra musical como instrumento al servicio de la construcción narrativa. Las páginas de Romain Rolland, E. M. Forster, Proust, James Weldon Johnson, Virginia Woolf, Aldous Huxley, Cadilhac, Palacio Valdés, Malraux o Thomas Mann nos servirán de guía en este recorrido interdisciplinar. 


\section{DE JEAN-CRISTOPHE (1904-1912) A AN A ROOM WITH VIEW (1908)}

El siglo XX se inició en Viena con el homenaje de los artistas de la Secesión en su XIV exposición a Beethoven, lejos del academicismo reinante. Se reúnen así en la misma sala el Friso a Beethoven de Gustav Klimt -como interpretación simbólica de la sinfonía coral, a través de sus tres paneles Anhelo de felicidad, Las fuerzas enemigas y La Música-, el último movimiento de la Novena interpretado por instrumentos de madera y latón bajo la dirección de Mahler, y la policromada escultural del genio, obra de Max Klinger ${ }^{1}$. Aquella pública manifestación representaba en el músico la encarnación de una divinidad que ofrecía su autosacrificio transformado en música como símbolo de un amor redentor. Esta representación plástica halló su correspondencia en la literatura que hizo de Beethoven el sujeto fascinante de toda fantasía artística. El premio nobel Romain Rolland escribió su Jean-Cristophe-novela de diez volúmenes publicada entre 1904-1912² - donde un compositor alemán -Jean-Christophe Krafft- es conformado con los sesgos del carácter de Beethoven y del propio novelista. Si bien sus primeros pasos vitales se aproximan a la vida del músico, según avanza la trama y el personaje envejece el narrador construye su psicología de un modo más independiente, sin renunciar eso sí a la condición del héroe que sublima su dolor a través de las fuerzas creativas, en una suerte de precedente del Adrian Leverkühn de Thomas Mann. A esta imagen de victimismo propiciado por un talento excepcional contribuyó igualmente la Vida de Beethoven del propio Rolland -traducida en España por Cernuda ${ }^{3}$, donde el escritor enfatiza en su semblanza el amor por la libertad y el heroísmo del genio de Bonn.

Paralelamente a esta empresa René Fauchois puso sobre la escena del Thêatre Odéon de París su Beethoven (1909) ${ }^{4}$, drama en tres actos que tuvo una extraordinaria acogida internacional, con treinta y cuatro personajes, nueve de los cuales son cada una de sus sinfonías, con quienes el compositor conversa y extienden un mensaje grandilocuente de esperanza, fe y victoria irónicamente preliminar al inicio de la Gran Guerra, un mensaje que se extendió en otras obras sobre la escena como el Beethoven Deaf (1911) de John Lawrence Lambe. En este mundo previo al fatal cataclismo apareció A Room with a View (1908), de E. M. Forster, la novela donde la joven Lucy Honeychurch se encuentra atrapada en un triángulo amoroso sofocante entre su prometido de clase alta y un joven socialista advenedizo que conoce casualmente en vacaciones. Su protagonista, pianista aficionada, descubre la verdadera disposición de sus intenciones siempre que se sienta al teclado y esta capacidad invocadora de la música es esencial para conocer el valor narrativo de su interpretación del primer movimiento de la sonata Op. 111. Como bien señala y no sin ironía uno de sus personajes, el Reverendo Beebe, "Si la señorita Honeychurch llega a vivir como toca, su vida

\footnotetext{
${ }^{1}$ Pintado el friso por Klimt en 1901, fue instalado en 1902 en el Pabellón de la Secesión, en Viena. Vid. más detalles en Koja (2006). Para los lectores en español vid. Hidalgo (2017, pp. 31-62). Más reciente es el trabajo de Jenkin (2019, p. 26 y ss).

2 Vid. Rolland (1904-1912), publicado en Cahiers de la Quinzaine, con las obras L'aube; Le Matin; L'Adolescent; La Révolte; La Foire sur la place; Antoinette; Dans la maison; Les Amies; Le Buisson ardent; La Nouvelle Journée.

${ }^{3}$ Vid. Rolland (1922). Existe la traducción de Luis Cernuda en Goethe y Beethoven (1934).

${ }^{4} \mathrm{Vid}$. Fauchois (1909). Fue escrita para el Teatro Odéon, con puesta en escena de André Antoine y la interpretación de Maxime Desjardins como Beethoven y Blanche Albane como Bettina). La obra fue traducida al inglés por Henry Grafton como Beethoven: A Dramatic Biography with an Accompaniment fo Beethoven's Music (1910). En Inglaterra la obra fue extraordinariamente popularizada por Sir Herbert Beerbohm Tree en el papel del compositor y Evelyn D'Alroy como Bettina Brentano (vid. Parker, 1928, pp. 388-395).
} 


\section{JUAN José PASTOR COMÍN}

será muy emocionante, tanto para nosotros como para ella" ${ }^{\text {" }}$. Lo cierto es que Beethoven despierta en el personaje una extraordinaria insatisfacción que necesita ser satisfecha en la búsqueda y entrega de lo novedoso. Más adelante en Howard's End (1910) dramatizará a través de la Quinta sinfonía el debate musicológico en torno a una estética de la recepción y el estatus de la música pura e instrumental ${ }^{6}$. En su capítulo V, con motivo del concierto en el Queen's Hall, se discutirá sobre las músicas de Beethoven, Brahms, Debussy y Wagner, con la explícita relegación de Edward Elgar, representante de la música del imperio. Paralelamente, las dos formas de escucha de la Quinta sinfonía que el narrador despliega a través de las diferentes actitudes de las dos hermanas, Helen y Margaret, nos revelará una dimensión política que la crítica ha visto como una respuesta a toda estética totalitaria -en una sorprendente anticipación-, bien pangermánica, bien decididamente imperialista.

\section{The Moonlight Sonata (1911), LA Symphonie Pastorale (1919) y À LA RECHERCHE DU TEMPS PERDU (1906-1922)}

Tras la Primera Guerra Mundial los nuevos nacionalismos y la instalación de un ambiente cínico, escéptico e iconoclasta, afectarán a la imagen estrictamente musical del compositor, tal y como evidencian los juicios de Debussy sobre un Beethoven que al mismo tiempo admira y critica en su Monsieur Croche ${ }^{7}$. Esta distancia ya pudo anticiparse en algunas novelas que llevaron al extremo una extravagante descripción del músico atormentado por su propio genio, tal y como sucede con obra de John Nordling The Moonlight Sonata (1911) ${ }^{8}$, asentada sobre la creencia de la dedicatoria de la Sonata Op. $27 n^{\circ} 2$ a la condesa Giulietta Guicciardi. No será así en el ámbito literario, donde el modernismo comenzará no tanto a servirse de la figura del compositor como a utilizar su música como medio técnico al servicio de la relación, bien de un modo experimental a través de una técnica contrapuntística, bien como instrumento para convocar a las voces interiores y monologantes de la novela lírica, tal y como de un modo excelente ha estudiado Nathan Waddell en Huxley, Wyndham Lewis, Dorothy Richardson, Rebecca West o Virginia Woolf (Waddell, 2019, pp. 90 y ss.)'.

Una de las obras beethovenianas cuyo título inspirará a novelistas y poetas será su Sinfonía pastoral, de la cual toma el título homónimo la novela de André Gide (La symphonie pastorale, 1919). Es singular que allí donde la música de Beethoven trata de ser elocuente a través de la representación sonora de la naturaleza, el núcleo temático de la novela sea la

\footnotetext{
5 "If Miss Honeychurch ever takes to live as she plays, it will be very exciting — both for us and for her." (Forster, 1908, p. 65).

${ }^{6}$ Sobre esta obra de Forster, vid. Zhou (2011, pp. 237-250) y Weatherhead (1985, pp. 247-264). Entre la abundante bibliografía, sin duda, el trabajo más completo sobre el lugar de Beethoven en la obra de E. M. Forster es el de Fillion (2010, en especial pp. 135 y ss.).

${ }^{7}$ Debussy fue extremadamente crítico con Beethoven. Si bien por un lado admiró la Novena Sinfonía, en otros lugares reserva juicios muy duros para composiciones como Adelaida, cuya melodía es un fragmento que «Le vieux Maître a dû oublier de brûler»; las últimas sonatas de piano adolecen de parecer transcripciones orquestales, o la Sinfonía Pastoral es condenada con sarcasmo como una obra pedante donde la escena de la tormenta es satirizada - «la frayeur que la nature inspire au coeur des hommes se perd dans les plis d'un liceul romantique», siendo Beethoven "responsable d'une époque où l'on ne voyait la nature qu'à travers les livres". (Debussy, ed. 1971, pp. 123 y ss.).

${ }^{8}$ Vid. Nordling (1912, ilustrada por Frank T. Merrill). Vid. Waddell (2019, pp. 89 y ss.).

${ }^{9}$ Consúltese además el trabajo colectivo editado por McParland (2009).
} 
ceguera física de Gertrude y la ceguera moral de pastor que adopta a esta joven huérfana asilvestrada dentro de un fatal triángulo amoroso que se complica cuando ella recobra la visión y se enamora de Jacques, hijo del pastor. Escrita a una edad ya madura, una vez traspasada la cincuentena, quizá como respuesta a un puritanismo opresor -no olvidemos que junto a otras de sus obras entró en 1952 en el Índice de libros probibidos del Vaticano-, la novela asemeja el modo en el que el anciano descubre a Gertrude el mundo con la forma en que la música revela los secretos de la naturaleza. Cuando Gertrude escucha, todavía ciega, la Pastoral, siente el anhelo de descubrir así la vida. Cuando recupera la visión, sin embargo, el referente del que cree estar enamorada no es el pastor, que la ha acompañado en sus descubrimientos entre las tinieblas -como si él mismo fuera la misma encarnadura musical-, sino el hijo de este. El fatal desenlace para todos los personajes -la locura, la desposesión, el exilio afectivo- semantiza el valor inaprehensible e impredecible del mensaje musical, capaz de suscitar los más insospechados referentes ${ }^{10}$.

Beethoven tiene igualmente un lugar preeminente en la escritura de Marcel Proust ${ }^{11}$ dentro de su $A$ la recherche du temps perdu ${ }^{12}$ (1906-1922), donde su música de cámara se convertirá en un eficaz motor narrativo ${ }^{13}$. En $A$ ' l'Ombre de jeunes filles en fleurs menciona cuatro de sus cuartetos, los números 12, 13, 14 y 15 $5^{14}$; del mismo Vinteuil se referencian su septeto y su sonata, incidiendo constantemente en el tiempo que necesitaron para su construcción como trasunto del que se requiere para la comprensión de una obra maestra, como lo fueron las últimas obras de cámara del alemán (Proust, ed. 1982, La Prisonnière, vol. 3, pp. 257). La obsesión de Proust por la música de cámara del alemán le llevó a que el cuarteto Poulet interpretara algunos de ellos en su casa en la primavera de 1916, al igual que sucede en su ficción con el Barón de Charlus (Proust, ed. 1982, Sodome et Gomorrhe, vol. 1, p. 751). La escritura en el Cuarteto n' 16 -“Muss es sein? / Es muss sein?"- juega en la obra un contrapunto singular con sus dudas y vacilaciones sobre su condición de escritor, tal y como sucede en $L a$ Prissonière (Proust, ed. 1982, vol. 3, pp. 250). La controversia sobre la petite phrase de Vinteuil -esa música que solo vive en la palabra- no está, como sabemos, en modo alguno agotada, pero buena parte de la crítica cree encontrar sobre ella, a tenor de las anotaciones del propio Proust, la ascendencia de la Sonata $n^{\circ} 32$ para piano, o, quizá de un modo más probable, el inicio del Cuarteto de cuerdas $n^{\circ} 12$ (también su Scherzo), o bien el Adagio del Cuarteto $n^{\circ} 8^{15}$.

\footnotetext{
10 Vid. Henry (1970, pp. 15-23). Consúltese igualmente el trabajo de Komur-Thilloy (2017).

${ }^{11}$ Entre los estudios más serios sobre la influencia de la música en Proust tenemos los de Matore \& Mecz, Irène (1982) y Nattiez (1987.

12 Escritas entre 1906 y 1922, lo siete tomos fueron publicados entre 1913 y 1927, los tres últimos tras la muerte del autor. Vid. Proust (ed. 1982, 3 vols.).

13 Vid. Shiomi (1997, pp. 150-162).

14 "Ce sont les quatuors de Beethoven (les quatuors XII, XIII, XIV,et XV) qui ont mis cinquante ans à faire naître, à grossir le public des quatuors de Beethoven, réalisant ainsi comme tous les chefs-d'oeuvre un progres sinon dans le valeur des artistas, du moins dans la societé des esprits, largement composée aujourd'hui de ce qui était introuvable quand le chef-d'oeuvre parut, c'est-à-dire d’êtres capables de l'aimer” (Proust, ed. 1982, vol. 1, pp. 343).

${ }^{15}$ Las implicaciones del valor de la música de Beethoven en Proust exceden, con mucho, la naturaleza de este trabajo que solo trata de presentar algunos icebergs sobre el gran océano de lecturas. Vid. Nattiez (1987, pp. 19 y ss.), Acquisto (2017, pp. 130 y ss); y Erickson (2009, pp. 160 y ss).
} 


\section{JUAN José PASTOR COMÍN}

\section{THE AUTOBIOGRAPHY OF AN EX-COLORED MAN(1912)}

Dentro igualmente del modernismo literario, pero desde la beligerante costa oeste del Atlántico, James Weldon Johnson, activista por los derechos humanos y figura destacada del renacimiento de Harlem, publicó The Autobiography of an Ex-Colored man (1912), un serio intento de demostrar que la igualdad racial podía alcanzarse a través de la expresión artística. Un narrador sin nombre cuenta su historia desde niño, criado solo por su madre, donde descubre en la escuela los prejuicios raciales sobre su condición de mulato. Educado musicalmente desde la infancia se ganará, una vez huérfano, la vida como intérprete incansable de ragtimes para blancos, especialmente para uno de ellos - un hombre rico cuya benevolencia despierta dudas sobre su propia identidad racial- que lo adoptará y lo llevará a Europa -viaje, según veremos, nada casual. En esta relación descubrirá las relaciones esclavo / maestro y decidirá disponer sus habilidades para la causa abolicionista. En París asistirá a una representación de Fausto y se sentará junto a una joven acompañada por sus padres y de quien se enamorará perdidamente. Sin embargo, descubrirá que el padre de ella es su propio padre y, ante el horror del incesto, huirá hacia el sur de Estados Unidos para profundizar en las formas musicales afro-americanas. Su visión, sin embargo, del linchamiento y quema de un negro le llevarán a renunciar públicamente a su condición -estrechamente vinculada a su interés musical- para ganarse la aceptación de los blancos. Se casará con una mujer a la que, tras un tiempo, revelará su condición y tendrá dos hijos a los que nunca les contará su origen racial. Obligado a vivir en una mediocridad degradada, su amigo negro de la infancia, Shiny, ofrece el contrapunto de la aceptación racial y triunfará como profesor en una universidad ayudando a la comunidad negra ${ }^{16}$.

James Weldon Johnson, como el anónimo protagonista de esta novela, fue a su vez un buen pianista y músico consumado, incapaz de tocar la Patética sin, como él mismo reconocía, la afectación de las alteraciones rítmicas propias del jazz y de las canciones sureñas de su infancia (vid. Kardos, 2009, pp. 126 y ss.). A nadie se le escapa que su protagonista se ofrece como un antihéroe en un contexto donde aún perduraba el recuerdo del Beethoven negro ${ }^{17}$, claro referente en la conformación del personaje. Nos referimos a la historia de Thomas Wiggins ("Blind Tom”, 1849-1908), conocido como Tom Bethune, el joven ciego y negro, de un oído portentoso, adoptado por el coronel esclavista James Green Bethune de Georgia y que fue explotado por su amo dado su increíble talento musical al piano, probablemente como consecuencia de un trastorno del espectro autista ${ }^{18}$. En 1860 llegó a tocar frente a James Buchanan en la Casa Blanca y en 1866 fue llevado a Europa y a los teatros ingleses, donde fue alabado por el mismo Ignaz Moscheles (O’Connell, 2009, pp. 168-171). Tras la muerte del coronel, su custodia fue ejercida por su esposa Elisa Stutzbach, quien poco después se divorciaría de él, aunque mantuviera los derechos sobre su custodia hasta su fallecimiento en 1908. Su figura, sin duda, todavía era recordada cuando Johnson publicó su novela: a pesar de vivir la Guerra de Secesión y atravesar la derrota de los estados esclavistas, su

\footnotetext{
16 Un análisis detallado de esta obra puede verse en la reciente publicación de Morrissette (2017) y Naviaux (2016, pp. 381-396).

${ }^{17}$ No nos centraremos aquí en el apodo "Schwarzspanier" que recibió el alemán y que ha dado lugar a una dudosa literatura, tanto científica como artística.

${ }^{18}$ Vid. O’Connell (2009), Grimsley (2008, pp. 1-11) y Smith (2016, pp. 164-175). Su legado está depositado en la Columbus State University.
} 
extraordinaria habilidad musical -consecuencia de su condición de músico savant- no le permitió esa emancipación hacia la libertad iluminada por el icono beethoveniano.

\section{Entre The Waves (1931), la Pastorale (1924), Sinfonía Pastoral (1932) y POINT COUNTER POINT (1928)}

Lejos de las luchas contra la segregación racial hallamos en el viejo continente a Virginia Woolf, a quien parte de la crítica atribuye la idea de que sus libros eran concebidos como música antes de ser escritos ${ }^{19}$ y que la estructura de obras como Mrs. Dalloway no pueden ser entendidas sin una visión que domine los elementos formales de la sonata o los recursos contrapuntísticos de la fuga ${ }^{20}$. Comprometida desde sus primeros ensayos con la realidad musical -"Street Music" (1905), "The Opera" (1906) o "Impressions of Bayreuth" (1909)- su satírica The Voyage Out (1915) nos ofrece en su protagonista Rachel Vinrace a una apasionada pianista aficionada que nos recuerda poderosamente a Lucy Honeychurch, la otra protagonstas de $A$ Room with a View, de E. M. Forster. Ambas interpretan la Sonata Op. 111 y se presentan muy conscientemente ante sus oyentes varones como elementos subversores de una musicología patriarcal que aleja de la mujer de este último periodo compositivo. Sus actitudes emancipatorias en ambas novelas suponen al mismo tiempo, según la crítica, una respuesta al pensamiento musicológico patriarcal que la música de Beethoven acumula, incluyendo a E.T.A. Hoffman, Adolf Bernard Marx, Richard Wagner, Romain Rolland, George Grove o Donald Francis Tovey (vid. Waddell, 2019, pp. 115 y ss. y Kelley, 2010, pp. 417-436).

La insatisfacción de Woolf con la novela y su voluntad de hacer de la palabra un elemento singular de resonancia personal y al mismo tiempo comunal y polifónica le llevan a encontrar en el discurso musical un modelo de construcción textual (Szegedy-Maszák, 2012, pp. 89-103). Ella misma confiesa en sus diarios que el monólogo final de Bernard en The Waves (1931) está comprometido por su escucha de los cuartetos de Beethoven ${ }^{21}$, los cuales fueron una constante en su vida ${ }^{22}$. De hecho, sus diarios nos refieren la escucha en el fonógrafo de sus sonatas durante el tiempo que precede a la escritura de The Waves. Ese tiempo de gestación coincide con la aparición en 1927 del conocido libro de J. W. N Sullivan sobre Beethoven, un autor al que la escritora conoció y cuya visión de los últimos cuartetos (Op. 130, 131 y la Gran Fuga Op. 133) Levi y otros autores que han seguido su estela han conciliado bien con el último monólogo de Bernard, quien compra una imagen del

\footnotetext{
${ }_{19}$ Así se lo reconoce a su amiga y violinista Elizabeth Trevelyan (1873-1956). Vid. Jacobs (1993, pp. 227-260) y Bazin (1973, p. 62).

${ }^{20} \mathrm{~V}$ id. el estudio clásico de Aronson (1980, pp. 21-31).

21 "It occurred to me last night while listening to a Beethoven quartet that I would merge all the interjected passages into Bernard's final speech, \& end with the words $O$ solitude: thus making him absorb all those scenes, \& having no further break. This is also to show that the theme effort, effort, dominates: not the waves: \& personality: \& defiance: but I am not sure of the effect artistically; because the proportions may need the intervention of the waves finally so as to make a conclusion" (Woolf, 1980 vol. 3, p. 339).

${ }^{22}$ En 1921 asistió a la integral de los mismos en el Beethoven Festival de Londres. (Woolf, 1978, vol. 2, pp. 14 y 114).
} 


\section{JUAN JOSÉ PASTOR COMÍN}

compositor ${ }^{23}$ mientras subsume todos los discursos en un juego contrapuntístico que asume líricamente del conjunto de voces que conforman el texto ${ }^{24}$.

La música de Beethoven, en consecuencia, constituyó un poderoso incentivo para renovar las fuerzas narrativas y supuso un aliento suficiente para la construcción de las conocidas como "novelas sinfónicas" - "roman d'expression symphonique"-, cuyos principios Paul-Émile Cadilhac, uno de los pilares en la redacción de L'Illustration, ya expuestos de facto en sus novelas Carnaval macabre (1919), L'Héroüque (1921) y, algo más tarde, en Les Flambeaux éteints ${ }^{25}$, desarrolla de un modo programático en el prólogo de su novela La Pastorale $(1924)^{26}$. Manifiestos de esta naturaleza han permitido desarrollar posteriormente una de las ramas de los estudios de literatura comparada más apasionante, el de las relaciones entre música y literatura ${ }^{27}$. Tomando así como modelos las sinfonías de Beethoven, Cadilhac sistematiza varios procedimientos de escritura musical: la asimilación sonora por el camino de la comparación o metáfora; conferir a los referentes sonoros una extrema viveza visual; crear deliberadamente un espacio de tensión entre lo oído -“estímulo percibido” - y lo escuchado -resultado de la percepción-; establecer un proceso de saturación tímbrica y sonora, acumulativo de los instrumentos precedentes; o el uso de los valores musicales lexicalizados como índices de importación patrimonial ${ }^{28}$. Señala así que en la base del "roman symphonique" hay tres tipos de temas:

\footnotetext{
${ }^{23}$ Veamos dos momentos reveladores de la novela. El primero de ellos sucede ante el retrato mismo de Beethoven. "Not that I love music, but because the whole of life, its masters, its adventurers then appeared in long ranks of magnificent human beings behind me; and I was the inheritor; I, the continuer; I, the person miraculously appointed to carry it on" (Woolf, 1931, p. 352). Más adelante: "Whatever sentence I extract whole and entire from this cauldron is only a string of six little fish that let themselves be caught while a million others leap and sizzle, making the cauldron bubble like boiling silver, and slip through my fingers. Faces recur, faces and faces -they press their beauty to the walls of my bubble- "Neville, Susan, Louis, Jinny, Rhoda and a thousand others. How impossible to order them rightly; to detach one separately, or to give the effect of the whole -again like music. What a symphony, with its concord and its discord and its tunes on top and its complicated bass beneath, then grew up!»" (Woolf, 1931, p. 354).

24 Vid. Levin (1983, pp. 164-171), Clements (2005, pp. 160-181) y Varga (2014).

${ }_{25}$ Nos referimos a sus obras Carnaval macabre, París: Fasquelle, 1919; L’Hérö̈que, París: Ferenzci, 1921; y Les Flambeaux éteints, París: Ferenzci, 1928. Vid. Ghil (2008, p. 251) y, muy especialmente, Stawiarski (2007, pp. 1$10)$.

${ }^{26}$ Vid. Cadilhac (1924, pp. 7-12). Veamos un ejemplo de "paysage musicalisée", donde el sonido trata de establecer una imagen: "L'andante du crépuscule, gris et mauve, emplissait la vallée et le ciel, s'élargissait mollement par grandes ondes calmes superposées. Les bas-fonds s'estompaient, laqués par places de noirs vigoureux trous d'ombre, masses boisées, rochers-, détachés comme des basses sur le dessin de l'orchestre. [...] Toute une gamme chromatique de couleurs éteintes - feuille morte, saumon, vieux ors, - chantait à l'octave supérieure, répétait le thème que de grands nuages, couleur de sang, reprenaient -très haut- avec des accents de cuivres. Des clochettes sonnaient comme des triangles et le grondement de la rivière, au fond de la gorge, prolongeait de lointains roulements de caisse et de timbales" (Cadilhac, 1924, p. 272). Vid. Schockley (2009, p. 86).

${ }^{27}$ Debemos, quizá, a su primer sistematizador en la segunda mitad del siglo XX a Brown (1948, p. 174).

28 Este texto puede representar adecuadamente los procedimientos referidos: "Cependant octobre avançait. Aux petites notes, haut piquées du début, au prélude mordant par des traits de flûte sur le final murmurant et mourant de l'été, succédaient des accords plus graves [...] : les frênes, les hêtres, les ormes, [...] se dépouillaient, perdaient sous la mousquetade de la pluie, les coups de vent lâchés au galop sur l'étendue désertique du causse, leurs feuilles jaunes, craquelées, mortes... Et les troncs apparaissaient, noirs et tortus, bossués de nœuds, semblables à des moignons, déviés comme des coxalgiques, mutilés par les émondages trop répétés, dressant, çà et là, des groupes d'infirmes, de grognards couturés par vingt batailles. Les bois [...] inscrivaient dans le paysage de grandes phrases dorées et somptueuses. Les bosquets de chênes [...] plongeaient le thème en point d'orgue avec leur feuillage mordoré qui persiste durant l'hiver [...]. Les haies d'églantiers [...] s'empourpraient avec des accents de cuivre, tandis que les pins des collines se réservaient [...] comme des orgues muettes" (Cadilhac, 1924, pp. 137-138). Vid. el desarrollo de estos procedimientos en Stawiarski (2015, pp. 127-148).
} 
Les uns très généraux, ayant une valeur de symbole, et traités en fugue (Dans La Pastorale: l'Amour, la Folie et la Mort aux prises avec les passions et la Vie); d'autres, secondaires, [...] correspondant au thème-varié des musiciens [...]; les derniers -véritables leitmotiveparticuliers aux personnages qu'ils caractérisent (Cadilhac, 1924, p. 9).

Esta forma de concebir musicalmente una novela fue desarrollada sistemáticamente en La Pastorale, donde los tiempos verbales son asimilados a la función de tonalidades y modos. El elemento paratextual de su título alude a Beethoven -aunque en realidad está en consonancia con el espacio campestre de la novela-, si bien el mismo Cadilhac confiesa que el último capítulo contiene una marcha fúnebre inspirada en la Eroica. Lejos de representar la estructura de la forma sonata en su primer movimiento, del scherzo o finale, el autor genera una forma autónoma literaria sobre los procedimientos de escritura contenidos en Beethoven (Brown, 1948, pp. 175 y ss.).

En la misma línea Armando Palacio Valdés (1853-1938), uno de los escritores menos conocidos de la Restauración, pero nominado dos veces al Premio Nobel, compañero de generación de Galdós, Clarín, Pardo Bazán o Pereda, publicó muy tardíamente su Sinfonía pastoral: novela de costumbres campesinas (1931), en la línea de la experimentación de Cadilhac, con la siguiente dedicatoria: "A la memoria imperecedera de Luis Beethoven, autor de la página musical más deliciosa que ha sonado hasta ahora en el mundo" ${ }^{29}$. La novela es un regreso a la naturaleza de Angelina, una joven melancólica y anoréxica que, para su sanación, aconsejado el padre por las autoridades eclesiásticas, es enviada a la aldea asturiana de Laviana con el pretexto de vivir con sus tíos en la paz del campo. En el tiempo que finge su progenitor recuperar la otra salud económica en La Habana, la joven se enamorará de Gustavo Manrique y recuperará su vitalidad. El autor utiliza igualmente cinco movimientos que alteran la disposición sinfónica original - Andante con moto; Adagio cantábile; Scherzo; Allegro ma non troppo y Presto finale-, en un intento por preservar cierto paralelismo, donde los subcapítulos, de gran brevedad, parecen imitar las intervenciones a solo instrumentales. El recurso al elemento epistolar acentúa el perspectivismo de la técnica compositiva y el texto, en su conjunto, constituye una evocación sentimental de la naturaleza en un intento de la novela por alcanzar la inmediatez y expresividad del texto musical admirado ${ }^{30}$.

Será, pues, en los años treinta cuando asistamos a una serie de creaciones experimentales en diferentes lenguas donde la música de Beethoven ocupará un lugar especial en la literatura universal. Dado que un recorrido pormenorizado por estas creaciones excede las dimensiones de este trabajo, nos detendremos en algunas de las obras más relevantes. Aldous Huxley constituye aquí un referente inexcusable: pianista consumado y notable crítico musical (Allis, 2013, pp. 13 y ss.), su novela Point Counter Point (London, Chatto \& Windus, 1928) supuso un deliberado esfuerzo por trasladar la escritura contrapuntística al ámbito narrativo. En su capítulo final uno de sus protagonistas, Spandrell, decide buscar una última prueba de la existencia de Dios mientras espera dejarse matar por los allegados de Everard

${ }^{29}$ Vid. Pérez Valdés (1931, p. 37)

30 Vid. Pérez Firmat (2017. pp. 1135-1149). 


\section{JuAn José Pastor Comín}

Webley, un político cuyo plan de asesinato había concebido y ejecutado. En los momentos preliminares al final trata de convencer a Mark Rampinon - otro de los protagonistas facetados, como perfiles de una misma melodía- de que el heiliger Dankgesang en modo lidio del Cuarteto para cuerdas n 15, Op. 132 en La menor de Beethoven es la "única prueba real de que Dios

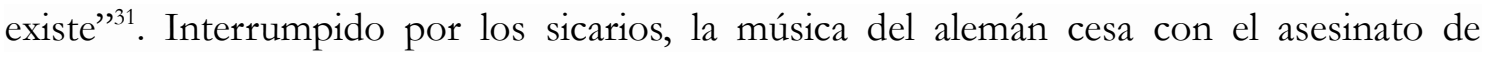
Spandrell, convirtiéndose la misma en un símbolo transfigurado del asesino rendido víctima $^{32}$. El lugar de Beethoven en la novela había sido preparado ya en el capítulo XXII donde se plantea la musicalización de la ficción desde los modelos de los Cuartetos Op. 130 y Op. 131, así como de las variaciones Diabelli:

The musicalization of fiction... But on a large scale, in the construction. Meditate on Beethoven. The changes of moods, the abrupt transitions (Majesty alternating with joke, for example, in the first movement of the $B$ flat major quartet. Comedy suddenly hinting at prodigious and tragic solemnities in the scherzo fo the CSharp minor quartet). More interesting still the modulations, not merely from one key to another, but from mood to mood. A theme is stated, then developed, pushed out of shape, imperceptibly deformed, until, though still recognizably the same, it has become quite different. In sets of variations the process is carried a step further. Those incredible Diabelli variations, for example. The whole range of thought and feeling, yet all in organic relation to a ridiculous little waltz tune. Get this into a novel. How? The abrupt transitions are easy enough. All you need is a sufficiency of characters and parallel, contrapuntal plots (Huxley, 1928, p. 408).

Algo similar sucede en el capítulo XXXIII, donde se expresa el contraste entre los Boborygmes symphoniques de Satie y la obertura Coriolán de Beethoven ${ }^{33}$. Esta técnica se extiende sin duda hacia su obra Eyeless in Gara (London: Chatto \& Windus, 1936), ejecutada sobre la construcción no diacrónica de la vida de Anthony Beavis, desde su infancia hasta su madurez (1890-1936). La obra traza un sinfín de referentes, desde el poema de John Milton sobre la condena de Sansón - de ahí procede su título-, hasta la yuxtaposición contapuntística de distintos periodos donde discurren algunos episodios autobiográficos, entre ellos las adicciones de su madre o su adhesión al pacifismo y al misticismo. Este camino beatífico de unión transcendental es reflejada por Huxley en la Missa Solemnis de Beethoven, especialmente en su Benedictus, donde subraya cómo se alcanza la unión y el entendimiento de la verdadera realidad (Huxley, 1931, pp. 44). Su escritura, en definitiva, no deja de reflejar al joven novelista que durante sus años de juventud entregó horas y horas al piano ${ }^{34}$ y que

\footnotetext{
${ }^{31}$ La presencia de este cuarteto tanto en Huxley como en Proust se entiende bien si, de acuerdo con las últimas interpretaciones musicológicas, le concedemos ese valor emancipatorio del tiempo. Vid. Theurer (2013, pp. 248-265).

32 Vid. Watt (1970, pp. 42-51) y May (1977, pp. 418-427).

33 Vid. Cupers (1985, p. 279)

34 “[...] we have just polished off the Beethoven Funeral March, having before that completed one of the variations in the sonata: I think that we are going to begin on a Chopin prelude next time-the one in $\mathrm{F}$ major, I think", en su carta a su padre, Leonard Huxley, el 16 de junio de 1912 (Huxley, 1969, p. 43). Uno de los mejore estudios sobre la temprana formación de Huxley como músico lo encontramos en Aplin (1983, pp. 2536).
} 
tanto en las obras arriba citadas como en su novela breve Two or Three Graces (1926) incluyó como elementos no solo referenciales sino esencialmente constructivos constantes referencias a las obras tardías de Beethoven ${ }^{35}$.

\title{
6. DE L'ESPOIR (1937) A DOKTOR FAUSTUS (1947)
}

Algunos escritores como André Malraux se servirán de la música como un elemento que expresa la visión interior de sus personajes frente al conflicto político ${ }^{36}$. Sucede así en el final de L'espoir (1937), una novela que recoge los eventos más importantes la Guerra Civil Española desde el levantamiento del 18 de julio hasta la victoria republicana en la batalla de Guadalajara, en marzo de 1937. La división tripartita del texto como si fuera una sinfonía clásica -L'illusion lyrique, donde se transmite el optimismo inicial de las fuerzas republicanas por detener el levantamiento; Le Manzanares, una oscura jornada de asedio a la capital y bombardeo de la Villa, aunque abierta a la esperanza; y, precisamente el último, L'espoir, un episodio frenético, como un presto finale que conduce a la victoria- tiene su sentido si atendemos a la condición de Manuel, su protagonista, un antiguo ingeniero de sonido extraordinariamente sensible a cualquier invocación de naturaleza musical. Vemos así sus reflexiones en el último capítulo de la novela mientras interpreta a Beethoven para sí mismo:

\begin{abstract}
Comme la musique supprimait en lui la volonté, elle donnait toute sa force au passé. Il se souvint du geste dont il avait tendu son revolver à Alba. Peut-ètre, comme le disait Ximénès, avait-il trouve sa vie. II etait né à la guerre, né à la responsabilité de la mort. Comme le somnambule qui soudain s'éveille au bord du toit, ces notes descendantes lui jetaient dans l'esprit la conscience de son terrible équilibre -"de l'équilibre d'où on ne tombe que dans le sang [...].. Il sentait la vie autor de lui, foisonnante de presages [...]. Un jour il y aurait la paix. Et Manuel deviendrait un autre homme, inconnu de lui-même, comme le combattant d'aujourd'hui avait été inconnu de celui qui avait acheté une petite bagnole pour faire du ski dans la Sierra [...] On ne découvre qu'une fois la guerre, mais on découvre lusieurs fois la vie [...] Ces mouvements musicaux [de Beethoven] qui se succédaient, roulés dans son passé, parlaient comme eût pu parler cette ville qui jadis avait arrêté les Maures, et ce ciel et ces champs éternels. Manuel entendai pur la première fois la voix de ce qui es plus grave que le sang des hommes, plus inquiétant que leur présence sur la terre: -la possibilité infinie de leur destin; et il sentait en lui cette presence mêlée au bruit des ruisseaux et aux pas des prisonniers, permanente et profonde comme le battement de son coeur (Malraux, 1937, pp. 359-360).
\end{abstract}

Los sufrimientos y heroicidades recogidas en sus páginas presentan, curiosamente su

\footnotetext{
35 Vid. Sounac (2002, pp. 305-310).

${ }^{36}$ Malraux mantuvo siempre una extraordinaria sensibilidad musical. En el homenaje de estado que recibió tras su muerte, presidido por Françoise Giraud, su sucesor en la Secretaría de Asuntos Culturales y Philippe de Gaulle, se escuchó el registro de su propia voz con los acordes de la Marche Funèbre de la sonata de Beethoven Op. 26 en Lab Mayor. Vid. Madsen (1976, p. 5). Consúltese al respecto el interesante artículo de Leavitt (1956, pp. 25-30).
} 


\section{JuAn José Pastor Comín}

trasunto en algunas ediciones sueltas de Beethoven ${ }^{37}$. En toda la obra la música y el canto son una constante que sugieren una confiada resistencia ante la amenaza totalitaria ${ }^{38}$. Resulta sin embargo significativo el hecho de que Manuel escuche en las páginas finales "des symphonies de Beethoven et les Adieux" en un espacio de irrealidad que, distanciándose de las anteriores evocaciones, parece convocar al abismo atroz y mortal de la guerra: "Comme le somnambule qui soudain s'éveille au bord du toit, ces notes descendantes et graves lui jetaient dans l'esprit la conscience de son terrible équilibre -de l'équilibre d'où on ne tombe que dans le sang” (Malraux, 1937, pp. 587-588).

Una novela excepcional surgida del exilio en la barbarie previa a la Segunda Guerra Mundial y que ha merecido una importante atención de la crítica es Doktor Faustus (Stockholm: Bermann-Fischer Verlag, 1947) de Thomas Mann, donde se ofrece una visión de la música absoluta desde la Sonata Op. 111, allí donde el pensamiento y el placer definitivamente se escinden ${ }^{39}$. El relato abarca la biografía completa de Adrian Leverkühn, un músico ficticio que pacta con el demonio veinticuatro años de una fulgurante carrera musical. Trasunto polifacético de Nietzsche, Schoenberg e incluso del mismo Beethoven, Adrian tiene un sobrino -Nepomuk- que morirá, al igual que Karl, el sobrino de Beethoven, en trágicas circunstancias. El tapiz de la novela es el desmoronamiento de Alemania con el ascenso nazi y la filosofía de Adorno se extiende por toda la obra. De hecho el mismo escritor reconoce en una carta dirigida a Adorno en octubre de 1943 su deuda con el filósofo al tomar para sí muchas de las ideas de su trabajo sobre el estilo tardío de Beethoven ${ }^{40}$, las cuales articulan prácticamente todo el capítulo VIII en el que tiene lugar la conferencia sobre la Sonata $\mathrm{n}^{\mathrm{o}} 32$ impartida por el Doctor Wendel Kretschmar, el inspirador y excéntrico musicólogo tutor de Leverkühn, que Mann construye sobre la figura del musicólogo alemán Hermann Kretzschmar (1848-1924), el cual revolucionó gracias a su concepto de "hermenéutica musical” el análisis musical al profundizar, más allá de las limitaciones de la estructura formal, sobre los aspectos discursivos de las obras ${ }^{41}$. La dependencia con respecto a Adorno llega a tomar, en ocasiones, el aspecto de un calco en la novela, tal y como se desprende en este fragmento de la conferencia de Kretzschmar:

\footnotetext{
${ }^{37}$ Sucede así con la siguiente edición: Souvennir à L. van Beethoven: six walses et une marche funèbre: pour le piano (París: Chez les fis de B. Schott, place des Italiens, $\left.\mathrm{n}^{\circ} 1,1828\right)$, que recoge el tercer movimiento de la sonata para piano Op. 26, transportada a La menor, y una compilación de valses de Schubert, extraídos de sus 36 Originaltänze, D. 365, entre los cuales se recogen los valses La douleur y L'espoir.

38 Vid. Viñals (2006, pp. 748). Su visión sigue muy de cerca a Morot-Sir (1989, pp. 178-187).

${ }^{39}$ Uno de los mejores trabajos que estudian las relaciones entre Thomas Mann y Adorno es el de Seiwert, E. (1995, pp. 123-200). Otros trabajos de obligada consulta son el estudio clásico de Engelberg, E. (1955, pp, 112116). A él hay que añadir Caro, E. E. (1959), Corbineau-Hoffmann, A. (1995, pp. 225-247), Gruchy (2019, pp. 195-208), Torabi, (2019, pp. 99-113) y Verster (1995).

40 Vid. Adorno (2003., pp. 114-126). Thomas Mann reconoce en su carta "una lectura estimulante y de mucha importancia para Kretzschmar, quien, demasiado inmerso en la historia de la música, se había quedado en la 'personalidad absolutizada', cuando él debía ser el hombre que llegara a la idea de que cuando se encuentran la muerte y la grandeza surge un objetivismo (con tendencia a la convención) en lo cual lo imperioso subjetivo se transforma en lo mítico. ¡No se asombre si Kretszchmar incorpora tales expresiones a su locuacidad!” (Adorno \& Mann, 2006, p. 9).

${ }^{41}$ Sobre esta importante figura de la musicología alemana vid. el interesante capítulo de Grimm (2000, pp. 8797).
} 
la relación de Beethoven con lo convencional aparece en las últimas obras de su vida, por ejemplo, en las últimas cinco sonatas para piano, como mucho más aflojada, menos vigilante. En estas obras tardías lo convencional, exento de desnudez, o si se quiere descarnado, desprovisto de individualidad y su majestuosidad ges más impresionante que la de cualquier atrevimiento personal. En esas obras, decía el orador, se establece entre lo subjetivo y lo convencional una nueva relación cuyo origen hay que buscarlo en la Muerte (Mann, 1947 [ed. 2009], p. 74) ${ }^{42}$.

La Arietta del Op. 111 se convierte, pues, en el corazón de la reflexión, influida por ese otro vals anodino y convencional de 1819, germen del colosal Op. 120, sus Variaciones Diabelli. Esta relación entre lo convencional y lo transcendente puede apreciarse en ambas obras, donde la iluminación entre las cinco variaciones de nuestra sonata y las treinta y tres de las Diabelli es clara, tal y como Pierre Brunel subrayó, al hallar cómo el tema de este segundo movimiento se despereza en la Variación XX de su última obra epigonal para el piano (Brunel, 1997, p. 136-137). El tratamiento de la forma, si bien no es despreocupado, sí se presta confiado a la fuerza cohesiva del tema, lo cual permite yuxtaponer cada variación bajo la coherencia de su patrón. Ahora bien, ¿cómo surge esta melodía? Adorno señaló que en Beethoven uno de sus medios formales más grandiosos era la sombra (Adorno, 2003, p. 70). Tras la umbría del primer trágico movimiento apenas se pronuncia una melodía parlante, enajenada de la convención y emancipadora de la opresión precedente que, como subjetividad, toma su ser de algo ajeno a sí misma. En el tema escrito en Do mayor y 9/16 sobre casi un ostinato de dominante, atendemos al ascenso melódico gradual y asimétrico - do, re, mi sol (c. 7)que se precipita en apenas tres compases hacia el final de la frase ${ }^{43}$. En su segunda frase la flexión hacia el sexto grado (cc. 9-12) es suspendida por el estatismo de la dominante en estado fundamental (c. 13), fuertemente expresivo en su total desnudez rítmica, armónica y melódica, y que abre una cadencia de acordes fundamentales para su conclusión (cc. 13-16) ${ }^{44}$.

A partir de este sencillo esquema se sucede la secuencia de variaciones: la primera transforma las corcheas con puntillo en juegos rítmicos sincopados de tres semicorcheas; la

\footnotetext{
42 Adorno escribe lo siguiente: "El arte siempre tiene su contenido meramente en el fenómeno. La relación de las convenciones con la subjetividad misma debe entenderse como la ley formal de la que emana el contenido de las obras tardías, en la medida en que éstas significan verdaderamente más que conmovedoras reliquias. Pero esta ley formal se hace patente precisamente en el pensamiento sobre la muerte" (Adorno, 2003, p. 115).

${ }^{43}$ La traducción española no recoge el homenaje que Mann hace a Adorno en la caracterización del tema de la arietta, omitiendo su cita específica: "Oigan las cadenas de trinos, los arabescos y las cadencias. Fíjense cómo lo convencional se impone. No se trata de eliminar del lenguaje la retórica, sino de eliminar de la retórica la apariencia de su dominio subjetivo. Se abandonan las apariencias del arte, el arte acaba siempre repudiando las apariencias del arte. ¡Dim-dada!” (Mann, 1947, ed. 2009, pp. 160-161). En realidad, en el texto en alemán dice lo siguiente: "Nach einem anlautenden $c$ nimmt es vor dem $d$ ein cis auf, so da $\beta$ es nun nicht mehr «Himmelsblau» oder «Wie-sengrund», sondern «O - du Himmelsblau», «Grü - ner Wiesengrund», «Leb’ - mir erwig wohl» skandiert; un dieses hinzukommende cis is die rührendste, tröstlichste, wehmütig versöhnlichste Handlung von der Welt” (Mann, 1947, p. 85). Esto tiene una difícil traducción que quizá no ha sido abordada con solvencia en nuestra lengua y que está comprometida por la escansión de largas y breves, y que tal vez pudiera ser así: «Después de un do inicial toma un do sostenido antes del re, por lo que ya no canta "cie-loazul" o "pra-dera", sino "oh, tu cieloazul", "Verde - pradera", "Me - despido"; y este do sostenido adicional es la acción más conmovedora, consoladora y sabiamente conciliadora del mundo».

44 Vid. Rosen (2006, pp. 299 y ss.).
} 


\title{
JuAn José Pastor Comín
}

segunda acelera el esquema anterior en dos grupos de semicorchea y fusa; la tercera anticipadoras de cierto sentido jazzístico - transforma el ritmo a cuatro grupos de semifusa y fusa, con sforzandi en partes débiles; la cuarta mantiene los ataques melódicos en tiempos débiles mientras la mano izquierda acompaña con el patrón uniforme de nueve fusas. Finalmente, el regreso a la melodía principal en la quinta variación -para algunos coda (cc. 160 y ss.)- supone la disolución de su dimensión temporal: el trino disuelve la articulación rítmica creciente, evoluciona hacia el trino triple -que le dio fama como joven concertista- y la inmovilidad sin medida se instala con toda la fuerza de una suspensión ${ }^{45}$. Críticos e intérpretes han encontrado en esta última conclusión la fuerza del que mira hacia atrás cuando se despide, donde la última mirada agranda lo vivido hasta lo desmesurado. La forma que concede Beethoven a este último movimiento altera la percepción de todo lo sucedido, de su propio pasado, y abandona a la música en un presente que ingresa en el silencio con el recuerdo de su memoria ya inalcanzable. Quizá por esta razón no debe extrañarnos la siguiente reflexión que Thomas Mann incorpora a la novela sobre la esencia del estilo tardío ${ }^{46}$ :

\begin{abstract}
El carácter distintivo de la frase es la gran separación entre el bajo y el distante, entre la mano izquierda y la mano derecha, y llega un momento, una situación extrema, en que el pobre motivo, solo y abandonado, parece flotar sobre un inmenso abismo, un instante de pálida sublimidad, seguido inmediatamente de un gesto de miedo, de espanto y de terror ante el hecho de que semejante cosa haya podido ocurrir. Pero muchas otras cosas suceden y se suceden antes de llegar al final. [...] El trillado motivo, que se despide de nosotros y se convierte él mismo en despedida, en un gesto y un grito de adiós, adquiere aquí una ligera ampliación melódica. [...] Es como si una mano amorosa nos acariciara el cabello o las mejillas, es como una última mirada clavada profundamente en nuestra pupila. Es como una bendición sobrehumana después de la terrible sucesión de formas violentas. Una despedida al oyente, un adiós eterno, de tan gran blandura para el corazón que arranca lágrimas a los ojos. Se cree estar oyendo palabras que dicen: «Olvida el tormento», «Todo fue sueño», «Dios es grande en nosotros», «No dejes de serme fiel.» Y de pronto todo se interrumpe [...] (Mann, 1947, ed. 2009, 76).
\end{abstract}

Y este parece ser el secreto esencial del $O p$. 111: el adiós de una música a sí misma que se sabe sin retorno. Thomas Mann abre así el camino hacia una novela de vanguardia apoyada sobre firmes cimientos musicológicos, y no sobre un cierto impresionismo musical, metafórico, o una representación bio-hagiográfica del compositor.

\footnotetext{
${ }^{45}$ Consúltese el interesante análisis de Verster (1999, pp. 4-23).

46 Conviene referenciar aquí entre los autores que, desde Adorno, han desarrollado este concepto de estilo tardío en Beethoven a Spitzer (2016, pp. 191-208) el cual se complementa muy bien con el trabajo de Bell (2016, pp. 131-145); consúltese también el libro de Said (2009, pp. 33 y ss.).
} 


\section{CONCLUSIONES}

Vemos, pues, cuáles han sido los usos que desde comienzos de siglo y hasta la fractura de la II Guerra Mundial recibió nuestro compositor. Existe, pues, una evolución desde el psicologismo de Romain Rolland y Marcel Proust hasta las propuestas más recientes. El tratamiento que se le concede a su obra y su persona recorre las críticas políticas hegemónicas de E. M. Forster; la denuncia del determinismo social en la novela de James Weldon Johnson; la dimensión de las relaciones humanas en el embrión familiar planteadas por Gide; el experimentalismo constructivo de Virginia Woolf y Cadilhac bajo dos prismas muy diferentes -la británica en el ámbito profundo de la estructura; el francés, en el espacio superficial de la dimensión sonora y eufónica-; la incardinación local de Palacio Valdés; la indagación psicológica de Aldous Huxley; la dimensión político-libertaria en la novela de Malraux y la última reflexión a dos voces que Thomas Mann nos presenta con Adorno como alter ego en su Doktor Faustus. Beethoven constituye así un elemento en la transformación del género narrativo, y se presenta instrumento útil para la configuración de los universos posibles en cada ficción. Sin duda sobre estas propuestas se desarrollarían, tras la deshumanización que en los años Cuarenta conmocionó a toda la humanidad, nuevos planteamientos que volverán a hacer de nuestro compositor un referente renovado en los nuevos discursos narrativos: ahí están las obras, en otro lugar analizadas por nosotros (Pastor, 2020b), de Alejo Carpentier, Ralph Ellison, Günter Grass, Anthony Burgess o Milan Kundera.

\section{BIBLIOGRAFÍA}

Acquisto, J. (2017). Proust, Music, and Meaning: Theories and Practices of Listening in the Recherche. Burlington: Palgrave Macmillan.

Adorno, Th. (2003). Beethoven. Filosofía de la música. Madrid: Akal.

Adorno, Th. \& Mann, Th. (2006). Correspondencia. 1943 - 1955. México: Fondo de Cultura Económica.

Allis, M. (2013). Temporaries and Eternals: The Music Criticism of Aldous Huxley, 1922-23. Cambridge: Cambridge Scholars Publishing,

Aplin, J. (1983). Aldous Huxley and Music in the 1920s. Music \& Letters, 64 (1-2), pp. 25-36.

Aronson, A. (1980). Music and the Novel: A Study in Twentieth Century Fiction. Totowa, N. J.: Rowman and Littlefield.

Bazin, N. T (1924). Virginia Woolf and the Androgynous Vision. New Brunskwick: Rutgerss University Press. 


\section{JuAn José Pastor Comín}

Cadilhac, P.-É. (1924). La Pastorale. París: Ferenczi et fils éditeurs.

Bell, M. (2016). Perceptions of Lateness: Goethe, Nietzsche, Thomas Mann and D. H. Lawrence. En G. McMullan \& S. Smiles (Eds.). Late Style and its Discontents. Essays in Art, Literature, and Music, pp. 131-145. Oxford: Oxford University Press.

Brown, C. S. (1948). Music and Literature: A Comparison of the Arts. Athens: University of Georgia Press.

Brunel, P. (1997). Les arpèges composés. Musique et Littérature. Paris: Klincksieck.

Capdepón Verdú, P. \& Pastor Comín, J. J. (eds.) (2020): Beethoven desde España: Estudios interdisciplinares y recepción musical (en prensa). Valencia: Tirant lo Blanch.

Caro, E. E. (1959). Music and Thomas Mann. Stanford: Stanford Honors Essays in Humanities, Number II.

Clements, E. (2005). Transforming Musical Sounds into Words: Narrative Method in Virginia Woolf's The Waves. Narrative, 133(2), pp. 160-181.

Corbineau-Hoffmann, A. (1995). Beethoven, Leverkühn und Thomas Manns Doktor Faustus. Arcadia, 39(3), pp. 225-247.

Cupers, J.-L. (1985). Aldous Huxley et la musique: à la manière de Jean-Sébastien... Bruselas: Publications des Facultés Universitaires Saint-Louis.

Engelberg, E. (1955). Thomas Mann's Faustu and Beethoven. Monatshefte, 47(2), pp. 112-116.

Erickson, G. (2009). Unstable Metaphors of Divinity: Proust's Theology and Musical Aesthetics. En R. McParland (Ed.) Music and Literary Modernism: Critical Essays and Comparative Studies, pp. 160-178. Cambridge: Cambridge Scholars Publishing.

Fauchois, R. (1909). Beethoven. Traducida al inglés por Henry Grafton como Beethoven: A Dramatic Biography with an Accompaniment fo Beethoven's Music [ed. 1910]. Nueva York: The New Theatre.

Fillion, M. (2010). Difficult Rythm. Music \& the Word in E. M. Forster. Illinois: University of Illinois.

Forster, E. M. (1908). A Room With a View. Londres: Edward Arnold.

Forster, E. M. (1910). Howards End. Londres: Edward Arnold.

Gerhard, A. (Ed.) (2000). Musikwissenschaft -eine verspätete Disziplin? Stuttgart: J. B. Metzler. 
Ghil, René (2008). De la poésie-scientifique \& autres écrits, Grenoble: ELLUG, Université Stendhal.

Gide, André (1919). La symphonie pastorale. París: Éditions de la Nouvelle Revue Française.

Grimsley, R. L. (2008). Discovering 'Blind’ Tom Wiggins: Creating Digital Access to Original Sheet Music at the Columbus State University Archives. Music Reference Services Quarterly, 10(2), pp. 1-11.

Gruchy, J. W. de (2019). The Search for Transcendence in an Age of Barbarism: Bonhoeffer, Beethoven, Mann's Dr. Faustus and the spiritual crisis of the present time. En M. Grebe (Ed.). Polyphonie der Theologie. Veranwortung un Widerstand in Kirche und Politik, pp. 195208. Stuttgart: Kohlhammer

Henry, F. G. (1970). La Symphonie Pastorale Revisited. Mountain Modern Language Association, 24(1), pp. 15-23.

Hidalgo, A. L. (2017). Lecturas hermenéuticas de la mujer en arte/comunicación/cultura. El caso del Friso de Beethoven, de Gustav Klimt. Escena. Revista de las Artes, 77(1), pp. 31-62.

Huxley, A. (1926). Two or Three Graces. London: Chatto \& Windus.

Huxley, A. (1928). Point Counter Point. London: Chatto \& Windus.

Huxley, A. (1931). Music at Night, London: Chatto \& Windus.

Huxley, A. (1936). Eyeless in Gaza, London: Chatto \& Windus.

Huxley, A. (1969). Letters of Aldous Huxley [edición de Grover Smith]. London: Chatto \& Windus.

Jacobs, P. (1993). 'The Second Violin Tuning in the Ante Room': Virginia Woolf and Music. En D. F. Gillespie (Ed.). The Multiple Muses of Virginia Woolf, pp. 227-260. Columbia: University of Missouri Press.

Jenkins, Ch. (2019). Ornament and the Vienna Secession: A Study of the 1902 Beethoven Exbibition. PhD Dissertation, Nueva York: City University of New York.

John, J. W. (1912). The Autobiography of an Ex-Colored Man. Boston: Sherman, French \& Company.

Kardos, M. (2009). Musical and Ideological Synthesis in James Weldon Johnson's The Autobiography of an Ex-Colored Man. En R. McParland (Ed.) Music and Literary Modernism: 


\section{JUAN José PASTOR COMíN}

Critical Essays and Comparative Studies, pp. 260-278. Cambridge: Cambridge Scholars Publishing.

Kelley, J. (2010). Virginia Woolf and Music. En M. Humm (Ed.). The Edinburgh Companion to Virginia Woolf and the Arts, pp. 417-436. Edinburg: Edinburgh University Press.

Koja, S. (Ed.) (2006). La destrucción creadora. Gustav Klimt, El Friso de Beethoven y la lucha por la libertad del Arte. Munich-Berlín-Londres-Nueva York: Prestel-Fundación Juan March.

Komur-Thilloy, G. \& Thilloy, P. (2017). André Gide, ou, L'art de la fugue: musique et littérature. Paris: Classiques Garnier.

Lambe, J. L. (1911). Beethoven Deaf. En Experiments in Play Writing. London: Sir Isaac Pitman \& Sons.

Leavitt, R. P (1956). Music in the Aesthetics of André Malraux. The French Review, 30(1), pp. 25-30.

Levin, G. (1983). The Musical Style of The Waves. The Journal of Narrative Tecnbique, 13(3), pp. 164-171.

Madsen, A. (1976) Malraux: A Biography. New York: Morrow.

Malraux, A. (1937). L'espoir. Paris: Gallimard "Folio".

Mann, Th. (1947). Doktor Faustus. Das Leben des deutschen Tonsetzsers Adrian Leverkühn, erzählt von einem Freunde, Stockholm: Bermann - Fischer Verlag [traducido en Doktor Faustus. Vida del compositor alemán Adrian Leverkühn narrada por un amigo. Barcelona: Edhasa, 2009].

Matore, G. \& Mecz, I. (1982). Musique et structure romanesque dans "A La recherche du tempsperdu". Paris: Klincksieck.

May, K. (1977). Accepting the Universe: The "Rampion-Hypothesis. "Point Counter Point and Island'. Studies in the Novel, 9(4), pp. 418-427.

McParland, R. P. (Ed.) (2009). Music and Literary Modernism: Critical Essays and Comparative Studies. Cambridge: Cambridge Scholars Publishing.

Morot-Sir, E. (1989). Visión de Goya, voix de Malraux dans L'espoir. Europe, 67(727), pp. 178-187.

Morrissette, N. (Ed.) (2017). New Perspectives on James Weldon Johnson's "The Autobiography of an Ex-Colored Man”. Athens: University of Georgia Press. 
Nattiez, J.-J. (1987). Proust Musicien, Paris: Christian Bourgois.

Naviaux, J. A. (2016). A Distinctly American Performance in James Weldon Johnson's The Autobiography of an Ex-Colored Man. CLA Journal, 59(4), pp. 381-396.

Nordling, J. (1912). Moonlight Sonata. Nueva York: Sturgis \& Walton.

O'Connell, D. (2009). The Ballad of Blind Tom, Slave Pianist: America's Lost Musical Genius. Nueva York: The Overlook Press.

Palacio Valdés, A. (1931). Sinfonía Pastoral: Novela de costumbres campesinas. Madrid: Victoriano Suárez.

Parker, C. (1938). René Fauchois. The French Review, 11(5), pp. 388-395.

Pastor Comín, J. J. (2020a). “... y la música se hizo verbo”. Imágenes poéticas de Beethoven. Valencia: Tirant lo Blanch (en prensa).

Pastor Comín, J. J. (2020b). Beethoven en los estantes: de la partitura a la ficción. Algunos apuntes para la construcción literaria del genio. En Capdepón, P. \& Pastor, J. J. (Eds.). Beethoven desde España: Estudios interdisciplinares y recepción musical (en prensa). Valencia: Tirant lo Blanch.

Pérez Firmat, G. (2017). Palacio Valdés, Untimely. Anales de la Literatura Española Contemporánea, 42(4), pp. 1135-1149.

Proust, M. (1982). À la recherche du temps perdu [édition établie et annotée par Pierre Clarac]. Paris: Gallimard (Bibliothèque de la Pléiade), 3 vols.

Rolland, R. (1904-1912). Jean-Christophe. Paris: Cahiers de la Quinzaine (L'aube; Le Matin; L'Adolescent; La Révolte; La Foire sur la place; Antoinette; Dans la maison; Les Amies; Le Buisson ardent; La Nouvelle Journée).

Rolland, R. (1934). Vie de Beethoven. Paris: Hachette [traducida por Luis Cernuda en Goethe y Beethoven. Madrid: Ediciones la Nave, nº 32, 1934].

Rosen, Ch. (2006). Las sonatas para piano de Beethoven. Madrid: Alianza Música.

Said, E. W. (2009). Sobre el estilo tardío. Música y literatura a contracorriente. Barcelona: Debate.

Schockley, A. (2009). Music in the Words: Musical Form and Counterpoint in the Twentieth-Century Novel. London \& New York: Routledge. 


\section{JuAn José Pastor Comín}

Seiwert, E. (1995). Beethoven-Szenarien. Thomas Manns "Doktor Faustus" und Adornos BeethovenProjekt. Stuttgart: J. B. Metzler.

Shiomi, H. (1997). Proust et les derniers quartuors de Beethoven. Études de langue et littérature françaises, 70, pp. 150-162.

Smith, W. W. (2016). 'Blind Tom' abroad: race, disability, and transatlantic representations of Thomas Wiggins. Journal of Transatlantic Studies, 14(2), pp. 164-175.

Sounac, F. (2002). Late Beethoven: deux scènes symboliques chez Huxley et Spender. Anglophonia / Caliban, (Musiques et littératures: Intertextualités), 11, pp. 305-310.

Spitzer, M. (2016). Notes on Beethoven's Late Style. En G. McMullan \& S. Smiles (Eds.). Late Style and its Discontents. Essays in Art, Literature, and Music, pp. 191-208. Oxford: Oxford University Press,

Stawiarski, M. (2007). Le sonore comme prétexte à image ou comme rater la musicalization du roman: l'exemple de Paul-Émile Cadilhac. En C. Rauseo, K. Zieguer \& A. Hutier (Eds.). Correspondances: vers une redéfinition des rapports entre la littérature et les arts, pp. 1-10. Valenciennes: Presses Universitaires de Valenciennes.

Stawiarski, M. (2015). Existe-t-il des métaphores musicales en littérature? En I. ThaillanderGuittard (Ed.). Métaphore et Musique, pp. 127-148. Rennes: Presses Universitaires de Rennes.

Sullivan, J. W. N. (1927). Beethoven: His Spiritual Development. New York: Knopf.

Szegedy-Maszák, M. (2012). Wagner or Beethoven? Shifts in the Musical Taste of Virginia Woolf. Neobelicon, 39(1), pp. 89-103.

Theurer, M. (2013). Playing with Time: the Heiliger Dankgesang and the Evolution of Narrative Liberation in Op. 132. Journal of Musicological Research, 32(2-3), pp. 248-265.

Torabi, J. (2019). Music, Myth and Modernity: From Nietzsche's The Birth of Tragedy to Thomas Mann's Doctor Faustus. Journal of Comparative Literature and Aesthetics, 42(1), pp. 99-113.

Varga, A. (2014). Virginia Woolf \& Music. Bloomington \& Indianapolis: Indiana University Press.

Verster, F. (1995). Language on Music: Beethoven, Mann and the Absolute (PhD. Doctoral Dissertation). Cape Town: University of Cape Town. 
Verster, F. (1999). Silence, Subjective Absence and the Idea or Ultimate Reality and Meaning in Beethoven's Last Piano Sonata, Op. 111. Ultimate Reality and Meaning, 22(1), pp. 4-23.

Viñals, C. (2006). André Malraux y Max Aub: dos visiones de la guerra civil española. En M. Bruña Cuevas et alii (eds.). La cultura del otro: español en Francia, francés en España, pp. 745754. Sevilla: Universidad de Sevilla.

Waddell, N. (2019). Moonlightning: Beethoven and Literary Modernism. Oxford: Oxford University Press.

Watt, D. J. (1970). The Criminal-Victim Pattern in Huxley's Point Counter Point. Studies in the Novel, 2(1), pp. 42-51.

Weatherhead, A. K. (1985). Howards End: Beethoven's Fifth. Twentieth Century Literature, 31(1-2), pp. 247-264.

Woolf, V. (1915). The Voyage Out. London: Ducworth \& Co.

Woolf, V. (1931). The Waves. London: The Hogarth Press [edición de New York: Harcourt, 1959].

Woolf, V. (1978). The Diary of Virginia Woolf, vol. 2, 1920-1924 [A. O. Bell, ed.]. New York: Harcourt.

Woolf, V. (1980). The Diary of Virginia Woolf. Vol. 3, 1925-1930 [A. O. Bell, ed.]. New York: Harcourt.

Zhou, M. (2011). Fiction as Musicology: Aesthetic Listening in E. M. Forster's Howard's End. Forum for Modner Language Studies, 47(3), pp. 237-250.

Fecha de recepción: 19/10/2020

Fecha de aceptación: 25/11/2020 